\title{
Immunogenicity and Safety of Concomitant Administration of 23- valent Pneumococcal Polysaccharide Vaccine and Trivalent Inactivated Influenza Vaccine in Adults Aged 50-70 Years
}

\author{
Yuanbao Liu \\ Jiangsu Province CDC: Jiangsu Province Center for Disease Control and Prevention \\ Ran Hu \\ Jiangsu Province CDC: Jiangsu Province Center for Disease Control and Prevention \\ Xiang Sun \\ Jiangsu Province CDC: Jiangsu Province Center for Disease Control and Prevention \\ Min Zhang \\ China National Biotec Group \\ Fenyang Tang \\ Jiangsu Province CDC: Jiangsu Province Center for Disease Control and Prevention \\ Lei Zhang \\ Jiangsu Province CDC: Jiangsu Province Center for Disease Control and Prevention \\ Xiaoqin Liu \\ china national biotec \\ Xuanwen Shi \\ china national biotec \\ Fubao Ma \\ Jiangsu Province CDC: Jiangsu Province Center for Disease Control and Prevention \\ Linyun Luo \\ china national biotec \\ Zhiguo Wang \\ Jiangsu Province CDC: Jiangsu Province Center for Disease Control and Prevention \\ Haiping Chen ( 15720801735@163.com ) \\ Centers for Disease Control and Prevention Division of High Consequence Pathogens and Pathology
}

\section{Research article}

Keywords: Influenza vaccine, Pneumococcal conjugate vaccine, Safety, Immunogenicity

Posted Date: November 4th, 2020

DOl: https://doi.org/10.21203/rs.3.rs-99542/v1

License: (1) (1) This work is licensed under a Creative Commons Attribution 4.0 International License. Read Full License 


\section{Abstract}

Background To explore the serological protective effect and to evaluate the safety after combined immunization with 23-valent pneumococcal polysaccharide vaccine and trivalent inactivated influenza vaccine in adults aged 50-70 years.

Methods This trial was designed as a single-center, randomized, single-blind, and positive parallel control vaccination study. A total of 1,065 people aged 50-70 years old were observed in Zhenjiang city, Jiangsu Province. Three groups were established, namely, the combined vaccine group (366 people), the pneumonia vaccine group (363 people) and the influenza vaccine group (364 people). The subjects were randomly assigned to each group.

Results A total of 1065 subjects were recruited. The noninferiority criterion of GMC ratios was met for all 7 pneumococcal serotypes after concomitant administration when compared with the administration of PPV23 alone. After concomitant administration, the noninferiority criterion of seroconversion rates was met for all influenza subtypes except the influenza $\mathrm{H} 3$ virus. The overall incidence of systemic adverse events was $6.67 \%$ (71/1065). The most frequently reported systemic adverse events were abnormal body temperature, muscle aches, nausea, and diarrhea. No statistically significant difference in systemic adverse events was found among the three groups.

Conclusion The local and systemic response rates in the combination group, pneumonia group and influenza group were similar to those of many similar vaccines, suggesting that the vaccine has good clinical safety. Serological findings from the combination vaccine suggest that it should be encouraged in the future to increase vaccination rates for influenza and pneumonia.

Trial Registration: ClinicalTrials.gov NCT02062281 February 13, 2014; Retrospectively registered.

\section{Introduction}

Influenza is an important contributor to mortality in elderly individuals worldwide. In urban China, during the period $2003-2008,86 \%$ of influenza-associated deaths occurred among people aged $\geq 65$ years ${ }^{[1]}$. Pneumococcal infections can also cause serious diseases, including meningitis, bacteremia, and pneumonia. Streptococcus pneumoniae is a common causative agent of pneumonia. Older adults, especially those older than 65 years, are thought to be a high-risk population for pneumococcal infections. The case fatality rates (CFRs) can be high, 15-20\% among adults and 30-40\% among elderly patients, even after appropriate antibiotic therapy and intensive care.

Therefore, the trivalent inactivated influenza vaccine (TIV) and 23-valent pneumococcal polysaccharide vaccine (PPV23) have been recommended to elderly people in many countries ${ }^{[2,5,6]}$. However, the coverage of TIV and PPV remains very low. Concomitant administration of TIV and PPV23 is considered one of the most effective ways to improve immunization coverage. To date, there have been some studies on the incidence of related diseases after pneumococcal polysaccharide vaccine and influenza vaccine combined, with reported improvements in respiratory symptoms. Studies on concomitant administration of TIV and PPV23 are still very limited ${ }^{[4-}$

7]. This study intends to evaluate the immunogenicity and safety of PPV23 and influenza virus split vaccine (TIV) at the same time in 50- to 70-year-old individuals and to provide a basis for the development of immunization strategies to prevent Streptococcus pneumoniae and seasonal influenza viral infections in elderly individuals.

\section{Methods And Methods}

\subsection{Study Design and participants}

This randomized, controlled, open-label study evaluated the safety and immunogenicity of PPV23 and TIV administered to healthy people as well as those with stable underlying diseases ( $\geq 8$ weeks) who were older than 50 years. The exclusion criteria were as follows: (1) a history of pneumonia in the previous 5 years, (2) previous pneumococcal vaccination in the past 5 years, and (3) previous influenza vaccination in the past year. Individuals with any known vaccine component allergies, any other vaccination or immunoglobulins within the previous 4 weeks, or a history of fever (axillary temperature $\geq 38^{\circ} \mathrm{C}$ ) within the previous 3 days were excluded. All the participants included were randomized in a 1:1:1 ratio into 3 groups: those receiving one dose of trivalent inactivated split-virus influenza vaccine (TIV, Group 1, 352 people); those receiving one dose of 23-valent pneumococcal polysaccharide vaccine (PPV23, Group 2, 353 people); and those concomitantly receiving one dose of split-virus influenza vaccine and 23-valent 
pneumococcal polysaccharide vaccine in different arms (TIV + PPV23, Group 3, 360 people) (see Fig. 1). The primary objective of the study was to demonstrate the differences in immune responses to influenza antigens or PPV23 serotypes 1 month after vaccination in the PPV + TIV group (concomitant administration) compared to those in the TIV group and PPV group separately. The secondary objective of the study was to assess the safety of concomitant administration in the PPV + TIV group.

\subsection{Vaccines}

PPV23 (Chengdu Institute of Biological Products Co., Ltd. Sichuan, China) contained the capsular polysaccharides of 23 Streptococcus pneumoniae serotypes $(1,2,3,4,5,6 \mathrm{~B}, 7 \mathrm{~F}, 8,9 \mathrm{~N}, 9 \mathrm{~V}, 10 \mathrm{~A}, 11 \mathrm{~A}, 12 \mathrm{~F}, 14,15 \mathrm{~B}, 17 \mathrm{~F}, 18 \mathrm{C}, 19 \mathrm{~A}, 19 \mathrm{~F}, 20,22 \mathrm{~F}, 23 \mathrm{~F}$ and $33 \mathrm{~F})$. Each $0.5 \mathrm{~mL}$ vial contained $25 \mu \mathrm{g}$ of purified polysaccharide from each serotype.

TIV (Chengdu Institute of Biological Products Co., Ltd. Shanghai, China) is an inactivated vaccine containing three influenza vaccine strains from the 2013-2014 Northern Hemisphere season: A/California/7/2009 (H1N1)pdm09-like virus, A/Victoria/361/2011(H3N2)like virus, and B/Wisconsin/1/2010-like virus.

\subsection{Procedures}

Blood samples $(4 \mathrm{~mL})$ were collected before the first administration (day 0 ) and at day 28 for immunogenicity evaluation. After centrifugation, the serum was frozen at $-20^{\circ} \mathrm{C}$ until being sent to laboratories for serological testing. The immune responses to the three antigens in the influenza vaccine were measured by using a standard hemagglutination inhibition (HI) assay. Seven pneumoniae serotypes $(1,2,5,6 \mathrm{~B}, 14,19 \mathrm{~F}, 23 \mathrm{~F})$ were chosen as the main indicators. The antibody level of the pneumonia vaccine was detected by ELISA.

Local or systemic reactions to the vaccines were monitored and recorded according to the guidelines for the classification of adverse reactions in vaccine clinical trials from the Food and Drug Administration. Injection-site adverse reactions (e.g., pain, redness, and swelling) and systemic adverse reactions (e.g., fever, diarrhea, and fatigue) were recorded during the 28 days postvaccination.

This study was conducted in accordance with the Declaration of Helsinki and Good Clinical Practice guidelines. The study was approved by the Medical Ethics Committee of the Jiangsu Provincial Centre for Disease Control and Prevention (NO: SL2015-B01502). The clinical protocol and informed consent forms were approved by the ethics committee of the Jiangsu Provincial Center for Disease Control and Prevention. All necessary documents were submitted to the ethics committee by the JSCDC. Each participant signed an informed consent form before being included in the study.

\subsection{Statistics analysis}

The mean geometric titers (GMT g/ml) of serum antibodies before and 1 month after immunization with PPV23 were calculated. The TIV positive conversion rate is defined as the proportion of those who were negative before GMC immunization and increased 1:40 or more after immunization or $\geq 4$ times after immunization. The cut-off value of the immunogenicity noninferiority evaluation of the seven serotypes of pneumococcus in the combined group was 0.5 , and the cut-off value of the noninferiority efficiency of the three serotypes of influenza was - 10\%. Quantitative data were calculated using a t test or an analysis of variance, and data that did not meet normality were tested using the Wilcoxon rank-sum test. The classification data were analyzed by the $\chi^{2}$-test or Fisher's exact probability method, and $P<0.05$ was considered statistically significant. All statistical analyses were performed using R3.5.1 software.

\section{Results}

\subsection{Baseline characteristics}

A total of 1065 subjects were recruited. The participants were randomly assigned in a 1:1:1 ratio into one of three vaccination groups: PPV + TIV (N = 360), PPV ( $N=353)$ and TIV ( $N=352)$. Baseline demographics were similar among the study groups (see Table 1). 
Table 1

Baseline characteristics of study subjects.

\begin{tabular}{|lllllll|}
\hline & & PPV + TIV $(\mathbf{N}=360)$ & PPV(N = 353) & TIV(N = 352) & F & p-value \\
\hline age & $(\mathrm{M} \pm$ STD $)$ & $60.0 \pm 4.7$ & $58.2 \pm 6.4$ & $57.81 \pm 5.2$ & 0.818 & 0.442 \\
\hline \multirow{2}{*}{ gender } & Male (\%) & $160(44.4)$ & $171(48.4)$ & $155(44.0)$ & 1.23 & 0.541 \\
\hline & Female (\%) & $200(55.6)$ & $182(51.6)$ & $197(56.0)$ & & \\
\hline
\end{tabular}

\subsection{Immunogenicity}

\subsection{Response to PPV}

Studies have already shown that the antibody GMC of the corresponding type of pneumonia was higher than that before immunization when PPV was administered alone.

Baseline GMCs of every serotype were not distinguishable between the PPV group and the PPV + TIV group (see Table 2). After PPV23 vaccination, GMCs increased markedly irrespective of concomitant administration of TIV. Overall, the comparison of geometric mean concentrations after 23-valent pneumococcal conjugate vaccine (PPV23) administration between the PPV group and the PPV + TIV group showed no significant differences (see Table 2). Consequently, the noninferiority criterion of GMC ratios was met for all 7 pneumococcal serotypes after concomitant administration when compared with the administration of PPV23 alone.

Table 2

Comparison of geometric mean concentrations after 23-valent pneumococcal conjugate vaccine (PCV23) administration: between PPV group and PPV + TIV group.

\begin{tabular}{|c|c|c|c|c|c|c|}
\hline \multirow[t]{2}{*}{ Pneumoniae serotypes } & \multicolumn{2}{|c|}{$\begin{array}{l}\text { Pre-Vaccination } \\
\text { GMC }\end{array}$} & \multirow[t]{2}{*}{ p-value } & \multicolumn{2}{|c|}{$\begin{array}{l}\text { Post-Vaccination } \\
\text { GMC }\end{array}$} & \multirow[t]{2}{*}{ p-value } \\
\hline & PPV group & PPV + TIV group & & PPV group & PPV + TIV group & \\
\hline 1 & 2.42 & 2.34 & 0.76 & 3.75 & 3.59 & 0.66 \\
\hline 2 & 2.12 & 2.18 & 0.45 & 3.98 & 3.91 & 0.54 \\
\hline 5 & 0.45 & 0.46 & 0.96 & 1.51 & 1.36 & 0.07 \\
\hline $6 B$ & 2.56 & 2.45 & 0.73 & 4.20 & 4.00 & 0.86 \\
\hline 14 & 7.24 & 6.54 & 0.06 & 13.30 & 12.62 & 0.62 \\
\hline $19 \mathrm{~F}$ & 2.34 & 2.32 & 0.90 & 4.31 & 4.00 & 0.49 \\
\hline $23 \mathrm{~F}$ & 1.72 & 1.68 & 0.35 & 3.71 & 3.36 & 0.35 \\
\hline
\end{tabular}

\subsection{Response to TIV}

GMCs increased significantly at one month postvaccination for all 3 serotypes irrespective of concomitant administration (see Tables 3 and 4). 
Table 3

Comparison of geometric mean concentrations after influenza vaccine administration: between TIV group and PPV + TIV group.

\begin{tabular}{|c|c|c|c|c|c|c|}
\hline \multirow[t]{3}{*}{ Subtype } & \multirow{2}{*}{\multicolumn{2}{|c|}{$\begin{array}{l}\text { Pre-Vaccination } \\
\text { GMC }\end{array}$}} & \multirow[t]{3}{*}{ p-value } & \multirow{2}{*}{\multicolumn{2}{|c|}{$\begin{array}{l}\text { Post-Vaccination } \\
\text { GMC }\end{array}$}} & \multirow[t]{3}{*}{ p-value } \\
\hline & & & & & & \\
\hline & TIV group & PPV + TIV group & & TIV group & PPV + TIV group & \\
\hline $\mathrm{H} 1$ & 15.67 & 16.80 & 0.77 & 319.37 & 298.46 & 0.41 \\
\hline $\mathrm{H} 3$ & 16.62 & 13.50 & 0.12 & 132.70 & 116.47 & 0.46 \\
\hline B & 50.66 & 73.04 & 0.06 & 476.32 & 526.32 & 0.72 \\
\hline
\end{tabular}

Table 4

Comparison of seroconversion rates between TIV group and PPV+TIV group (a. antibody titer $\geq 10$, b. antibody titer $\geq 40$ ).

\begin{tabular}{|lllllllll|}
\hline Influenza serotypes & TIV & PPV+TIV & $\chi^{2}$ & P-value & TIV & PPV+TIV Post- & $\chi^{2}$ & P-value \\
& Pre- & Pre- & & & Post- & & \\
H1 & $73.30 \%$ & $76.82 \%$ & 1.17 & 0.28 & $100.00 \%$ & $100.00 \%$ & - & - \\
\hline H3 & $69.89 \%$ & $65.08 \%$ & 1.88 & 0.17 & $100.00 \%$ & $96.37 \%$ & 13.25 & 0.00 \\
\hline B & $96.31 \%$ & $98.04 \%$ & 1.96 & 0.16 & $100.00 \%$ & $100.00 \%$ & - & - \\
\hline
\end{tabular}

b.

\begin{tabular}{|lllllllll|}
\hline Influenza serotypes & TIV & PPV+TIV & $\chi^{2}$ & P-value & TIV & PPV+TIV Post- & $\chi^{2}$ & P-value \\
& Pre- & Pre- & & & Post- & & \\
\hline H1 & $24.43 \%$ & $25.70 \%$ & 0.15 & 0.70 & $98.86 \%$ & $97.49 \%$ & 1.88 & 0.17 \\
\hline H3 & $28.69 \%$ & $19.83 \%$ & 7.67 & 0.01 & $90.63 \%$ & $85.20 \%$ & 4.93 & 0.03 \\
\hline B & $69.89 \%$ & $80.73 \%$ & 11.36 & 0.00 & $98.86 \%$ & $98.88 \%$ & 0.01 & 0.98 \\
\hline
\end{tabular}

In this study, for the influenza $\mathrm{H} 1$ virus, the seroconversion (titer $\geq 40$ ) rates were $98.86 \%$ for the TIV group and $97.49 \%$ for the PPV + TIV group; for the H3 virus, rates were $90.63 \%$ for the TIV group and $85.20 \%$ for PPV + TIV group; for the B virus, rates were $98.86 \%$ for the TIV group and $98.88 \%$ for the PPV + TIV group. After concomitant administration, the noninferiority criterion of seroconversion rates was met for all influenza subtypes except the influenza $\mathrm{H} 3$ virus.

\subsection{Safety}

Approximately 85 of 1065 participants were reported to have adverse events. The majority of adverse events were mild. The overall incidence of local adverse events was $1.31 \%$ (14/1065). The most common local reactions were pain at the injection site, cough and swelling. Pain was more common in the TIV group ( $1.57 \%$ vs. $0.69 \%$ for the PPV + TIV group and $0.29 \%$ for the PPV group, $P<0.001)$.

The overall incidence of systemic adverse events was $6.67 \%$ (71/1065). The most frequently reported systemic adverse events were abnormal body temperature, muscle aches, nausea, and diarrhea. No statistically significant difference in systemic adverse events was found among the three groups.

The incidence of adverse reactions in the adult group was 34.00\% (85 persons), the incidence of grade 1 adverse reactions was $30.00 \%$ (75 persons), and the incidence of grade 2 adverse reactions was 3.60\% (9 persons); grade 1 adverse reactions accounted for $88.24 \%$ 
(75/85), and grade 2 adverse reactions accounted for $10.59 \%(9 / 85)$ of the total number of reactions. In addition, a grade 3 adverse reaction occurred in the adult group (see Table 5).

Table 5

Classification of adverse reactions after immunization

\begin{tabular}{|c|c|c|c|c|c|c|c|c|c|c|c|c|}
\hline \multirow[t]{2}{*}{ Group } & \multirow{2}{*}{$\begin{array}{l}\text { Number } \\
\text { of } \\
\text { observers }\end{array}$} & \multicolumn{4}{|c|}{ First order reaction } & \multicolumn{4}{|c|}{ Secondary reaction } & \multicolumn{3}{|c|}{ Tertiary reaction } \\
\hline & & $\mathrm{N}$ & $\begin{array}{l}\text { Composition } \\
\text { ratio }\end{array}$ & $\chi^{2}$ & $P$ & $\mathrm{~N}$ & $\begin{array}{l}\text { Composition } \\
\text { ratio }\end{array}$ & $x^{2}$ & $P$ & $\mathrm{~N}$ & $\begin{array}{l}\text { Composition } \\
\text { ratio }\end{array}$ & $\chi^{2} \quad P$ \\
\hline $\begin{array}{l}\text { PPV } \\
+ \text { TIV }\end{array}$ & 360 & 16 & $4.44 \%$ & 7.076 & 0.029 & 5 & $1.39 \%$ & 1.887 & 0.389 & & & \\
\hline PPV & 353 & 33 & $9.35 \%$ & & & 2 & $0.57 \%$ & & & 1 & $0.28 \%$ & \\
\hline TIV & 352 & 26 & $7.39 \%$ & & & 2 & $0.57 \%$ & & & & & \\
\hline
\end{tabular}

\section{Discussion}

The annual influenza epidemic causes millions of cases of severe illness and thousands of deaths worldwide[2]. Moreover, influenza is considered to be an important factor in high winter rates of pneumococcal pneumonia during nonpandemic periods in some studies [10-12], showing the advantage of simultaneously preventing pneumococcal infections and influenza. Vaccination is the most effective strategy to prevent both influenza and pneumococcal infections. Unfortunately, even several years after these vaccines are licensed for humans, the coverage rates are still not high. In China, the pneumococcal vaccine coverage rate in older adults is approximately $1 \%$. Even in some resource-rich countries, pneumococcal vaccine coverage rates are also relatively low, with rates of approximately $20-40 \%$. Considering the low vaccine coverage rates, concomitant influenza and pneumococcal vaccination has been recommended as an effective measure to increase pneumococcal vaccine coverage in both children and elderly individuals.

Epidemiological data show that the serotype of pathogenic pneumococci is different domestically and abroad. Currently, the 7-valent pneumococcal conjugate vaccine currently used in China does not contain the first few serotypes, i.e., 5, 1, 2, 3, 6A, 15. The main pneumococcal serotypes isolated in different regions of China are $6 \mathrm{~A}, 6 \mathrm{~B}, 19 \mathrm{~F}$, and $23 \mathrm{~F}$. The Newmo method instructions suggest that serotypes that often cause drug-resistant pneumococcal infections are 6B, 9V, 14, 19A, 19F, 23F. In this study, 7 serotypes $(1,2,5,6 \mathrm{~B}$, $14,19 \mathrm{~F}$ and $23 \mathrm{~F}$ ) were selected on the basis of relevant literature for the detection of protective antibody levels after pneumococcal polysaccharide vaccination.

In this study, immunogenicity results showed that immune responses for TIV antigens after the concomitant administration of PPV23 and TIV to elderly individuals are robust and noninferior to immune responses after TIV given separately. In addition, there were no statistically significant differences for any of the seven serotypes in GMCs after concomitant administration of PPV23 and TIV compared with the administration of PPV23.

Similarly, in some foreign countries, studies have shown that concomitant administration of influenza vaccine and PPV23/PCV13 resulted in acceptable immunogenicity and safety when compared to either agent given alone[4, 5, 7, 13]. A randomized, controlled trial in the United States found that the anti-pneumococcal IgG responses were statistically significantly lower for 8 of 13 serotypes after concomitant administration of PCV13 and TIV than after the administration of PCV13 alone [4]. A retrospective study conducted by Japanese scientists found that, compared with people who received only the influenza vaccine, those who received a coadministration of pneumococcal and influenza vaccines had lower the rates of respiratory infections[14]. For adults, especially those aged $\geq 65$ years, studies in both Italy and Sweden supported the efficacy of concomitant administration[15, 16]. A study in Italy suggested that the administration of influenza and pneumococcal vaccines leads to substantial health benefits and to a reduction in mortality[15]. The prospective study in Sweden also showed that rates of hospitalization declined and mortality decreased in the vaccinated cohort[16].

Concomitant vaccination had no additive effects on adverse events in this study. A study from South Korea showed that, compared with the TIV group, local adverse reactions and some systemic adverse events were more common in the PPV group or the PPV + TIV group[7, 17].

Our analysis is subject to several limitations. First, the period of observation was not long enough because it was limited to one influenza season. However, our sample size could counteract this limitation to some degree. Second, we did not consider whether there 
was any difference in results based on different injection sites. Some previous studies have performed research on this topic but found no difference.

In conclusion, concomitant administration of PPV23 and TIV did not show significant interference in antibody responses and demonstrated good safety profiles. Although the defects of the current vaccines cannot be ignored, biomedical research on vaccines will enable many individuals to live longer, more comfortable lives. In addition, it will help to avoid draining health resources and straining the social welfare system.

\section{Conclusions}

Overall, The local and systemic response rates in the combination group, pneumonia group and influenza group were similar to those of many similar vaccines, suggesting that the vaccine has good clinical safety.

\section{List Of Abbreviations}

1.trivalent inactivated influenza vaccine (TIV)

2.23-valent pneumococcal polysaccharide vaccine (PPV23)

3.Geometric antibody titres (GMCs)

4.Enzyme-Linked Immunosorbent Assay (ELISA)

\section{Declarations}

\section{Ethics approval and consent to participate}

Not applicable.

\section{Consent for publication}

Not applicable.

\section{Availability of data and materials}

The data used and analyzed for the current study is available upon request from the first author Yuanbao Liu (E-mail:

94724252@qq.com).

\section{Competing interests}

Not applicable.

\section{Funding}

This work was supported by the National Natural Science Foundation of China under Grant number 81502860; the Jiangsu Provincial Medical Youth Talent under Grant number QNRC2016547; the "333" Project of Jiangsu Province under Grant number BRA2017538.

\section{Availability of data and materials}

All authors had full access to the data and materials. Data are available within this article. Detailed data is available from the corresponding author upon reasonable request.

\section{Authors' contributions}

Conceived and designed the experiments: RH, LZ, YL. Performed the experiments: MZ, FT. Analyzed the data: LZ, WM, YL. Contributed reagents/materials/analysis tools: XL. Wrote the paper: HP, FM, XS. All authors read and approved the final manuscript.

\section{Acknowledgements}


We thank the staff of the local counties' Centers for Disease Control, who spent numerous hours and effort working with us in obtaining, verifying, and cleaning up the data used in this study.

\section{References}

1. Song JY, Jin CH, Yeon HJ, Yun NJ, Bin SY, Seon KI, et al. Outpatient-Based Pneumococcal Vaccine Campaign and Survey of Perceptions about Pneumococcal Vaccination in Patients and Doctors. Yonsei Medical Journal. 2013;54:469-75.

2. Song JY, Cheong HJ, Hyun HJ, Seo YB, Lee J, Wie SH, et al. Immunogenicity and safety of a 13-valent pneumococcal conjugate vaccine and an MF59-adjuvanted influenza vaccine after concomitant vaccination in $\geqslant 60$-year-old adults. Vaccine. 2016;35.

3. Dagan R, Poolman J, Siegrist CA. Glycoconjugate vaccines and immune interference: A review. Vaccine. 2010;28:5513.

4. Jr FR, Gurtman A, Rubino J, Smith W, Van CM, Jayawardene D, et al. Randomized, controlled trial of a 13-valent pneumococcal conjugate vaccine administered concomitantly with an influenza vaccine in healthy adults. Clinical \& Vaccine Immunology Cvi. 2012;19:1296.

5. Harris AM. Use of 13-valent pneumococcal conjugate vaccine and 23-valent pneumococcal polysaccharide vaccine among children aged 6-18 years with immunocompromising conditions: recommendations of the Advisory Committee on Immunization Practices (ACIP). Mmwr Morbidity \& Mortality Weekly Report. 2013;62:521.

6. Schwarz TF, Schmoele-Thoma B. Assessment of functional antibacterial opsonophagocytic antibodies elicited by 13-valent pneumococcal conjugate vaccine administered concomitantly with trivalent influenza vaccine in a randomized clinical trial in adults aged $\geq 65$ years. Vaccine. 2013;31:291.

7. Song JY, Cheong HJ, Tsai TF, Chang HA, Choi MJ, Jeon JH, et al. Immunogenicity and safety of concomitant MF59-adjuvanted influenza vaccine and 23-valent pneumococcal polysaccharide vaccine administration in older adults. Vaccine. 2015;33:4647-52.

8. Destefano F, Goodman RA, Noble GR, Mcclary GD, Smith SJ, Broome CV. Simultaneous Administration of Influenza and Pneumococcal Vaccines. Journal of the American Medical Association. 1982;247:2551-4.

9. Zhu F, Xu W, Xia J, Liang Z, Liu Y, Zhang X, et al. Efficacy, safety, and immunogenicity of an enterovirus 71 vaccine in China. New England Journal of Medicine. 2014;370:818-28.

10. Walter ND, Taylor TH, Shay DK, Thompson WW, Brammer L, Dowell SF, et al. Influenza Circulation and the Burden of Invasive Pneumococcal Pneumonia during a Non-pandemic Period in the United States. Clinical Infectious Diseases. 2010;50:175-83.

11. Kim PE, Musher DM, Glezen WP, Rodriguezbarradas MC, Nahm WK, Wright CE. Association of invasive pneumococcal disease with season, atmospheric conditions, air pollution, and the isolation of respiratory viruses. Clinical Infectious Diseases An Official Publication of the Infectious Diseases Society of America. 1996;22:100.

12. Pasi P, Liselotte H, Katarzyna G, Åke S, Karl E. Occurrence of invasive pneumococcal disease and number of excess cases due to influenza. Bmc Infectious Diseases. 2006;6:58.

13. Durando P, Rosselli R, Cremonesi I, Orsi A, Albanese E, Barberis I, et al. Safety and tolerability of 13-valent pneumococcal conjugate vaccine in the elderly. Human Vaccines. 2015;11:172-7.

14. Sumitani M, Tochino Y, Kamimori T, Fujiwara H, Fujikawa T. Additive inoculation of influenza vaccine and 23-valent pneumococcal polysaccharide vaccine to prevent lower respiratory tract infections in chronic respiratory disease patients. Internal Medicine. 2008;47:1189-97.

15. Christenson B, Lundbergh P, Hedlund J, Örtqvist A, Ke. Effects of a large-scale intervention with influenza and 23-valent pneumococcal vaccines in adults aged 65 years or older: a prospective study. Lancet. 2001;357:1008.

16. Grilli G, Fuiano L, Biasio LR, Pregliasco F, Plebani A, Leibovitz M, et al. Simultaneous influenza and pneumocococcal vaccination in elderly individuals. European Journal of Epidemiology. 1997;13:287-91.

17. Scott LJ, Sanford M. Pneumococcal Polysaccharide Conjugate Vaccine (13-Valent, Adsorbed). Drugs. 2012;29:847-55.

\section{Figures}




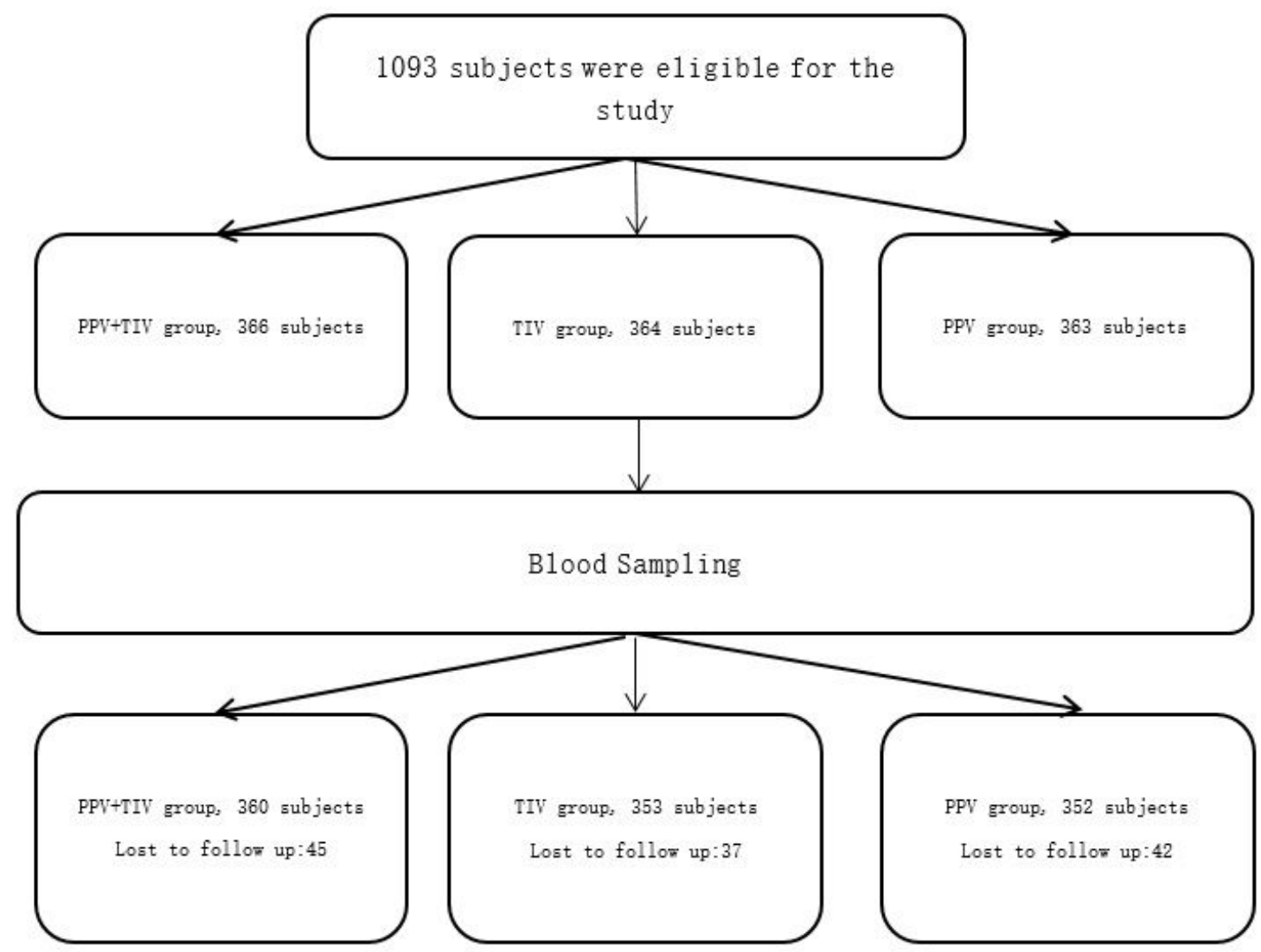

Figure 1

Study Design and participants

\section{Supplementary Files}

This is a list of supplementary files associated with this preprint. Click to download.

- CONSORT2010Checklist.doc 